\title{
First report of deinonychosaurian trackway from the Cretaceous
}

\section{of Guizhou, China}

\author{
Yanjiao Qin ${ }^{a}$, Lida Xing ${ }^{b}{ }^{*}$ \\ a Geological Museum of Guizhou, Guiyang 550081, China; \\ b State Key Laboratory of Biogeology and Environmental Geology, China University of Geosciences, \\ Beijing 100083, China. Corresponding author. Lida Xing: xinglida@gmail.com \\ ' School of the Earth Sciences and Resources, China University of Geosciences, Beijing 100083, China.
}

(C) The Authors, 2021

\begin{abstract}
The southern edge of Sichuan Basin has a long-standing folklore about the

Tian Ji Stone, which actually tells of the theropod tracks. Here we describe

a new Tian Ji track site named Xinglongwan in Chishui, Guizhou Province,

China. Two kilometers away from the old site recorded in 2011, the later with cf. Irenesauripus isp. are morphologically different from the Xinglongwan theropod tracks. The tridactyl tracks from Xinglongwan site have been assigned to the cf. Eubrontes. Didactyl tracks in the Xinglongwan site, which are the first discovery of deinonychosaurian tracks in Guizhou Province, are assigned to the Velociraptorichnus. Both tracks were recorded in the report about ichnofauna in Jiaguan Formation, representing the diversity of theropod tracks in Sichuan Basin. The authors also briefly discuss the preservation mode and potential external-morphological changes of cf. Eubrontes and Velociraptorichnus from Xinglongwan site.
\end{abstract}

ARTICLE HISTORY

Received: 28-12-2020

Revised: 18-03-2021

Accepted: 29-05-2021

\section{KEYWORDS}

Eubrontes

Velociraptorichnus

Sichuan Basin

Jiading Group

Jiaguan Formation

Wotoushan Formation

\section{Introduction}

A large collection of tetrapoda tracks have been found in the Jurassic-Cretaceous strata in Sichuan Basin (Xing et al., 2016a). Residents, whose ancestors originated in Baoyuan town, Chishui, Guizhou Province, have their own explanation to the footprints on the huge rocks laying on the mountains: Tian Ji (Heavenly Chicken) tracks, due to their shapes of chicken footprints. The origin of this legend is unknown, but similar footprints and legends have appeared in Sichuan and Guizhou provinces, providing an example of vertebrate tracks contributing to the formation of myths and legends (Xing et al., 2011a).

Xing et al (2011b) described seven dinosaur trackways in Baoyuan. The tracks were preserved on the red thick feldspar and quartz-sand layer of Jiaguan Formation, and were provisionally assigned to cf. Irenesauripus isp.. Many of the tracks contained extended metatarsal pads. The vast dinosaur tracks discovered in Jiaguan Formation have almost become the only channel for us to learn about the Cretaceous vertebrate fauna in Sichuan Basin. This fauna is dominated by Saurischia tracks, which indicates the rich diversity of theropod (Xing \& Lockley, 2016).

In 2006, unidentified marks on the collapsed rocks in Xinglongwan during the construction of a road in Pinghua Village, Baoyuan Township, Chishui City, were thought to be the local legendary Tian Ji tracks (Fig. 1). Later in 2020, The Guizhou Geological Museum transported 
these specimens to the museum in Guiyang as a permanent exhibit (catalogued as GP0101-1). Xinglongwan site is a landscape of forests, with no dinosaur tracks being found in the initial stratum. The tracks are all on the surface of the slumped rock, and the levels are generally accompanied by developed desiccation cracks. Xinglongwan site is located about $2 \mathrm{~km}$ southwest of Baoyuan site and preserves morphologically inconsistent tracks, which we describe briefly here.

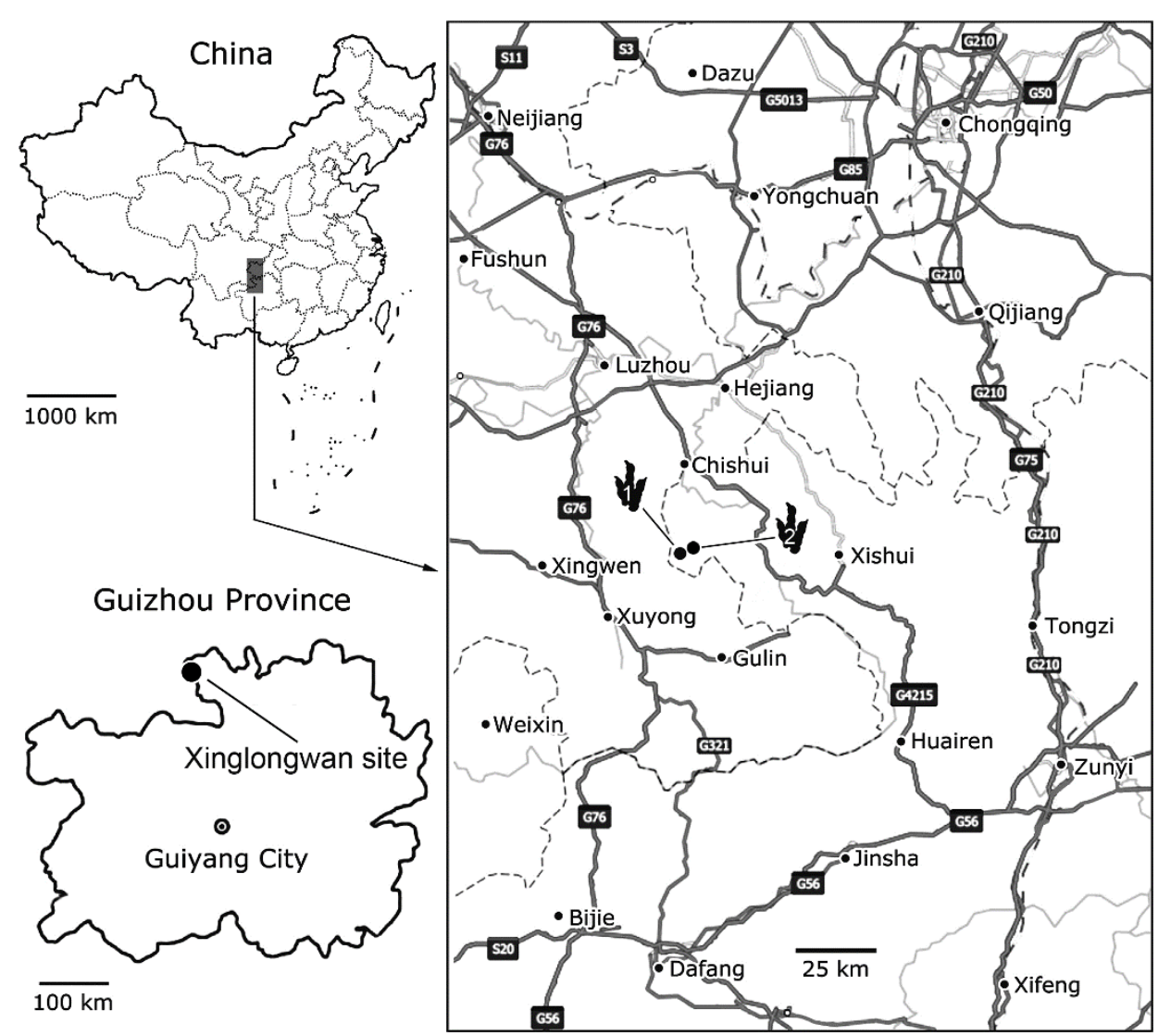

Figure 1. Map showing the location of Xinglongwan site (1) and Baoyuan site (2) in the Guizhou Province, China.

\section{Geological setting}

Chishui City is located at the southern margin of the Sichuan Basin, where a 3,000-4,000 m thick red clastic construction and interbedded sandstone and mudstone were deposited during the Jurassic to Cretaceous. The delineation of this set of red clastic rocks is controversial. According to the division scheme of Yibin Subdivision in Sichuan Basin (Long et al., 2011), this set was, from bottom to top, divided into the Lower Cretaceous Wotoushan Formation, the MidCretaceous Da'erdan Formation, the Upper Cretaceous Sanheguan and Gaokanba formations, and the Paleocene Liujia Formation. The lower part of Da'erdan Formation and the Wotoushan Formation can be equated with the Jiaguan Formation of the Chengdu-Ya'an Division in Sichuan Basin.

In the stratigraphic division scheme of Guizhou Province, the Cretaceous-Paleocene strata was referred to the Jiading Group and was divided from bottom to top into the Upper Cretaceous Wotoushan Formation, the Lower Cretaceous Sanhe Formation, and the Paleocene Liujia Formation. The Jiading Group is the main formation in Chishui City, with an outcrop area of $1346.57 \mathrm{~km}^{2}$ (Leng et al., 2017), and the lithology is brown-purple and brick-red quartz sandstone interspersed with dark purple-red mudstone, about $942 \mathrm{~m}$ thick, which is at the margin of the basin (Hao et al., 2000), mainly distributed in the south of Chishui River in Chishui City. The mainly stratigraphic distribution of the Chishui Danxia landform is the Wutoushan and Sanhe formations.

The Sanhe Formation is equated to the Upper Member of the Jiaguan Formation. It is an interbedded brick-red thin layer to medium-thick layered muddy feldspar sandstone and brick-redpurple mudstone. The Wotoushan Formation is equated to the Lower Member of the Jiaguan Formation, which is a brick red thick layer to massive feldspar quartz sandstone with a very 
small amount of brick red mudstone and pisolitic limestone (Wang, 2020). Sandstone has cross bedding, and sometimes huge oblique bedding can be seen to several meters. Mud cracks, ripple marks or raindrop impressions are also seen on mudstone and siltstone layers. The bottom sandstone contains $2-3.5 \mathrm{~m}$ thick conglomerate, which is pseudo-integrated on the purple-red mudstone and sandstone of the Late Jurassic Penglaizhen Formation.

The Jiading Group is mainly composed of sandstone interbedded with mudstone, with strong resistance to weathering. Tall ridges, mostly covered by vegetation, are mainly formed in this terrain. Vertical joints and fissures are developed in the sandstone layer. Under the influence of temperature difference and weathering, the cliff area is prone to collapse (Leng et al., 2017). Exposed strata within the Xinglongwan area belong to the upper section of
Cretaceous Jiaguan/Sanhe Formation, and is primarily formed by medium-thick to hugelythick feldspar-clastic sandstone interbedded with thin mudstone. The area from Xishui (Guizhou Province) to Hejiang of Luzhou City was a braided river deposit in the Cretaceous (Geng, 2011).

\section{Morphology}

The length and width of GP0101-1 are $2.56 \mathrm{~m}$ and $1.8 \mathrm{~m}$ respectively, and it is divided into two layers with developed mud cracks (Fig. 2). At least 14 footprints are distributed on the layer of Lay 1, including 12 tridactyl tracks and 2 didactyl tracks. All tracks are preserved as natural casts (convex hyporeliefs). Most of the tracks are covered by mud cracks, indicating that the tracks are left before the desiccation.

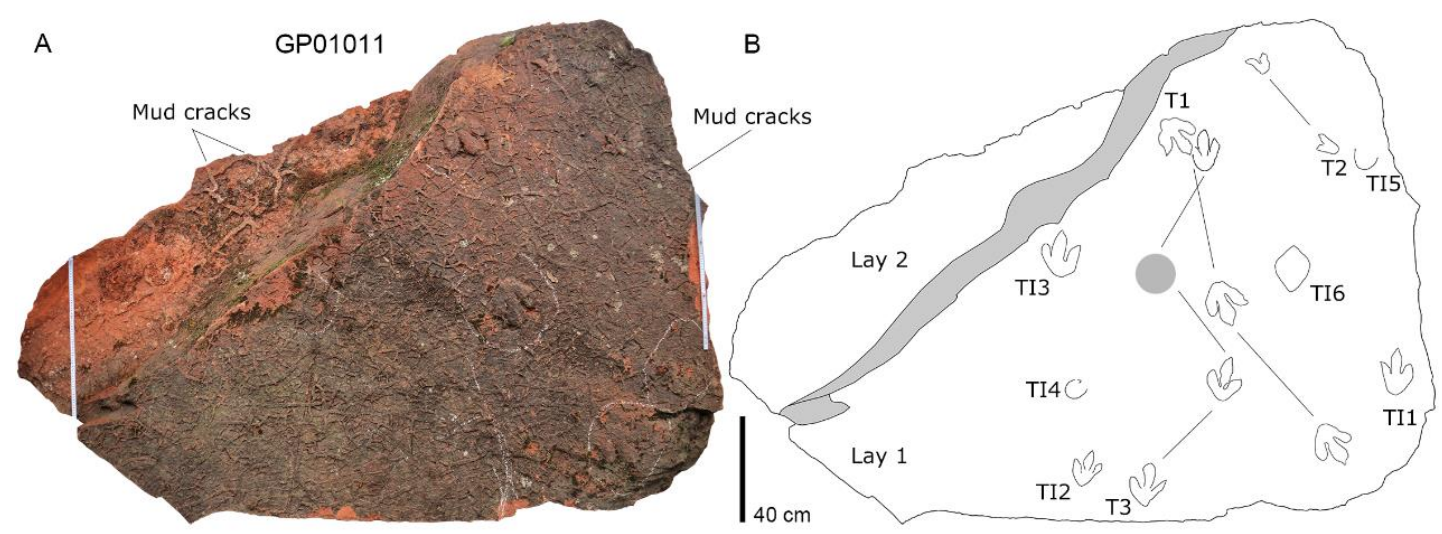

Figure 2. Photograph of the GP0101-1 specimen from Xinglongwan site, Guizhou Province, China.

\subsection{Didactyl tracks}

Material: Two natural casts of didactyl pes impressions, cataloged as GP0101-1-T2-R1 and L1 (Figs. 2, 3, Table. 1) from the Xinglongwan site.

\begin{tabular}{c|cccccccccc}
\hline GP0101-1- & L & W & PL & SL & PA & II-III & III-IV & II-IV & L/W & M \\
\hline T1-R1 & 19.0 & 15.0 & 65.0 & 127.0 & 152 & 33 & 33 & 66 & 1.3 & 0.39 \\
T1-L1 & 18.5 & 16.0 & 66.0 & - & - & 35 & 25 & 60 & 1.2 & 0.47 \\
T1-R2 & 18.3 & 13.5 & - & - & - & 28 & 31 & 59 & 1.4 & 0.49 \\
Mean & 18.6 & 14.8 & 65.5 & 127.0 & 152 & 32 & 30 & 62 & 1.3 & 0.45 \\
T2-R1 & 9.7 & 5.6 & - & - & - & - & 48 & - & 1.7 & - \\
T2-L1 & 9.3 & 7.3 & 40.0 & - & - & - & 35 & - & 1.3 & - \\
Mean & 9.5 & 6.5 & 40.0 & - & - & - & 42 & - & 1.5 & - \\
T3-R1 & 17.5 & 12.5 & 48.0 & - & - & 28 & 30 & 59 & 1.4 & 0.38 \\
T3-L1 & 16.5 & 12.5 & - & 85.0 & - & 29 & 31 & 60 & 1.3 & 0.62 \\
T3-R2 & - & - & - & - & - & - & - & - & - & - \\
T3-L2 & 17.0 & - & - & - & - & - & 34 & - & - & - \\
Mean & 17.0 & 12.5 & - & 85.0 & - & 29 & 32 & 60 & 1.4 & 0.50 \\
TI1 & 18.0 & 11.5 & - & - & - & 24 & 26 & 50 & 1.6 & 0.57 \\
T12 & 13.5 & 9.5 & - & - & - & 30 & 24 & 54 & 1.4 & 0.45 \\
T13 & 18.5 & 13.8 & - & - & - & 28 & 29 & 57 & 1.3 & 0.44 \\
TI6 & 17.5 & 11.5 & - & - & - & & & & 1.5 & - \\
\hline
\end{tabular}

Table 1. Measurements (in $\mathrm{cm}$ and ${ }^{\circ}$ ) of the dinosaur tracks from Xinglongwan tracksite, Guizhou Province, China. Notes: Abbreviations: L: Maximum length; W: Maximum (measured as the distance between the tips of digits II and IV); PL: Pace length; SL: Stride length; PA: Pace angulation; II-III, IIIIV, II-IV: angle between digits II-III, III-IV, II-IV; L/W is ML/MW's dimensionless; M: Mesaxony. 

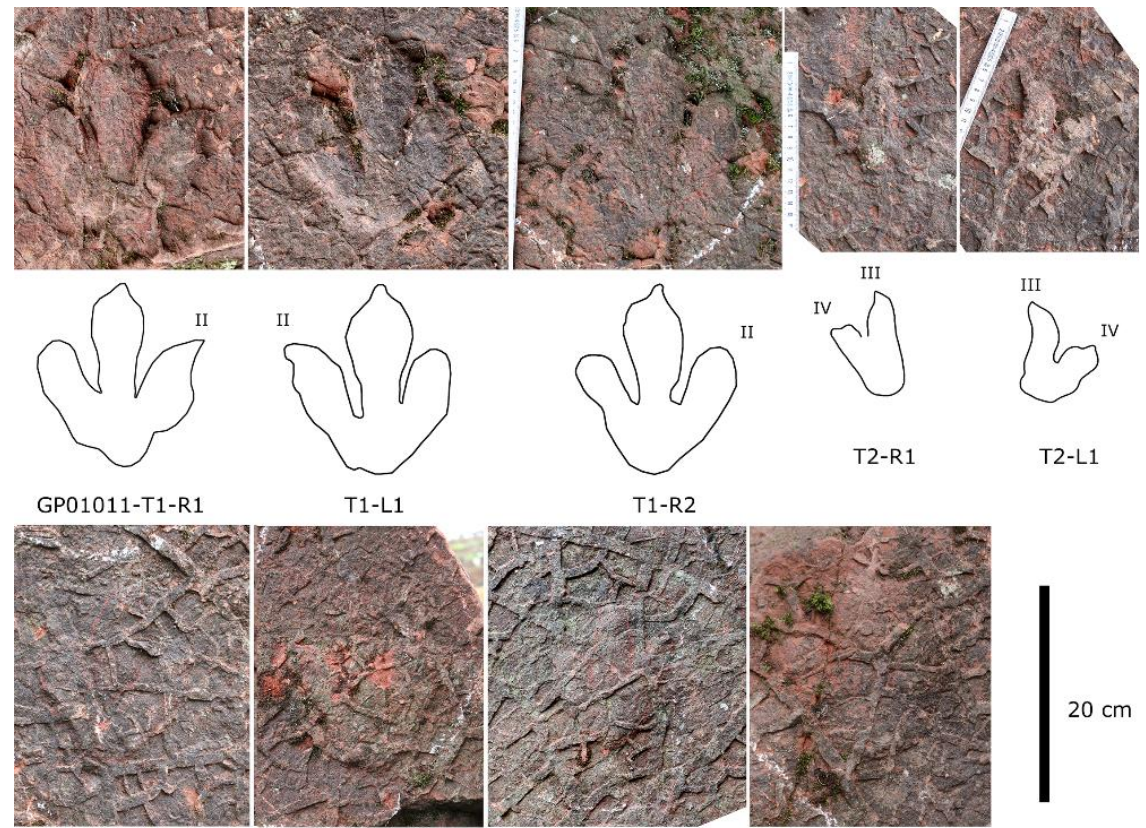

T1-L1

T1-R2
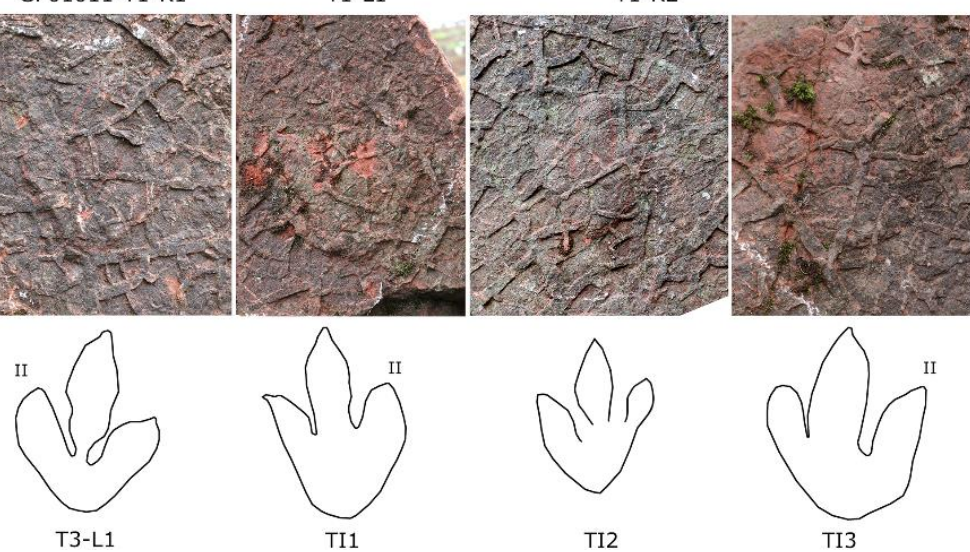

Figure 3. The photograph and interpretative outline drawings of GP0101-1 theropod tracks.

Description: GP0101-1-T2-R1 and L1 are the single pace with a mean length/width ratio of 1.5 . The pes tracks are elongate (average length 9.5 $\mathrm{cm}$ ) with two-digit impressions (digits III and IV) and rounded heel. The impressions of digits III are longer than digit IV, and roughly equal in width. The average divarication angle between digits III and IV of GP0101-1-T2 is $42^{\circ}$. Claw impressions are relatively sharp. The large metatarsophalangeal region is semicircular and not separated from the digit traces by a distinct border. No trace of a digit II impression is visible in either track. GP0101-1-T2-R1 and L1 are interpreted as a right-left step of $40 \mathrm{~cm}$.

Comparisons and discussion: The didactyl pes imprints with the absence of digit II traces in all specimens indicate their affinity to deinonychosaurs. Deinonychosaurian ichnotaxa currently consists of five ichnogenera (Dromaeopodus, Dromaeosauripus, Menglongipus, Sarmientichnus and Velociraptorichnus) (Kim et al., 2008, 2012; Xing et al., 2013a, b, 2018a, b). Velociraptorichnus is characterized by relatively robust digits, and by the absence of well-defined digital pads, or digit curvature, all features shared by the Xinglongwan tracks. The Xinglongwan didactyl tracks and Velociraptorichnus sichuanensis are similar in size and morphology. But there is minor difference between them. For instance, the divarication angle between digits III and IV in Xinglongwan specimens is slightly larger than the Velociraptorichnus holotype, but almost as same as the Mujiaowu Velociraptorichnus isp. Therefore, we refer the didactyl tracks from the Xinglongwan tracksites tentatively to Velociraptorichnus isp.

The table 2 shows all records of Velociraptorichnus so far, demonstrating some interesting phenomena. In terms of morphological consistency, tracks with a length of 7-11 cm have a stable ratio $(4.2-5)$ between pes length and track length. The divarication angle between digit III and IV has a larger range, which is from $27^{\circ}$ to $42^{\circ}$. In all of the 7 records, three sites were found in Jiaguan Formation (Barremian-Albian), two sites were from Xiaoba Formation (the First Member of the Xiaoba Formation is equivalent to the Jiaguan Formation from the Sichuan Basin, CGCMS, 1982), with two sites respectively located in Tianjialou Formation (Barremian-Albian, Kuang et al., 2013) and the Third Member of the Tuchengzi Formation (Late Jurassic Oxfordian-Tithonian or Early Cretaceous Neocomian, or BerriasisanBarremian). These records widespread in Barremian, corresponding to the paleoecological pattern proposed by Lockley et al. (2013): Dromaeopodus, Velociraptorichnus, Minisauripus and Eubrontes tracks, also bird tracks (Koreanornis) with distinct East Asian features. 


\begin{tabular}{|c|c|c|c|c|c|c|}
\hline & $\begin{array}{c}\text { Site, } \\
\text { Province }\end{array}$ & Fm. & $\begin{array}{l}\text { Length } \\
\text { (cm) }\end{array}$ & III-IV & PL/L & References \\
\hline V. sichuanensis & $\begin{array}{l}\text { Emei, } \\
\text { Sichuan }\end{array}$ & Jiaguan & 11.0 & $30^{\circ}$ & 5.0 & $\begin{array}{l}\text { Zhen et al., } 1994 \\
\text { Xing et al., 2013b }\end{array}$ \\
\hline Velociraptorichnus isp. & $\begin{array}{l}\text { Junan, } \\
\text { Shandong }\end{array}$ & Tianjialou & $\sim 10$ & $27^{\circ}$ & - & Li et al., 2007 \\
\hline$V$. zhangi & $\begin{array}{l}\text { Mujiaowu, } \\
\text { Sichuan }\end{array}$ & Xiaoba & $8.5-10.4$ & $36^{\circ}$ & - & Xing et al., 2015 \\
\hline Velociraptorichnus isp. & $\begin{array}{l}\text { Mujiaowu, } \\
\text { Sichuan }\end{array}$ & Xiaoba & 11.0 & $41^{\circ}$ & 4.2 & Xing et al., 2015 \\
\hline cf. Velociraptorichnus & $\begin{array}{l}\text { Shimiaogou, } \\
\text { Sichuan }\end{array}$ & Jiaguan & 7.1 & $37^{\circ}$ & 4.6 & Xing et al., 2016b \\
\hline Velociraptorichnus isp. & $\begin{array}{l}\text { Chengde, } \\
\text { Hebei }\end{array}$ & Tuchengzi & 8.7 & $34^{\circ}$ & 4.2 & Xing et al., 2019 \\
\hline Xinglongwan specimens & $\begin{array}{l}\text { Xinglongwan, } \\
\text { Guizhou }\end{array}$ & Jiaguan & 9.5 & $42^{\circ}$ & 4.2 & This paper \\
\hline
\end{tabular}

Table 2. Cretaceous Velociraptorichnus records from China. Notes: Abbreviations: Fm: Formation; IIIIV: angle between digits III-IV; PL/L: Pace length/maximum length

\subsection{Tridactyl tracks}

Material: 12 natural casts of tridactyl pes impressions, cataloged as GP0101-1-T1, T3, and 6 separated tracks (Figs. 2, 3, Table. 1). The two trackways are each composed of three tracks, while $\mathrm{T} 3$ is missing a right footprint.

Description: The theropod trackway GP0101-1T1 is consists of three tridactyl footprints preserved as natural casts (convex hyporeliefs), labelled GP0101-1-T1-R1-R2 (Figures 2-3) (Table 1). The footprints have an average length and width of $18.6 \mathrm{~cm}$ and $14.8 \mathrm{~cm}$, respectively. The average length: width ratio of the imprints is 1.3 , the pace angulation $152^{\circ}$ and the average divarication angle between digits II and IV $62^{\circ}$. One step $(65 \mathrm{~cm})$ is about 3.5 times the length of a single footprint. GP0101-1-T1-R1 is the bestpreserved track in the GP0101-1-T1 trackway. In GP0101-1-T1-R1, digit II is the shortest, and digit III is slightly shorter than digit IV. The digital pads in three digits are unclear due to preservation. Claw marks in digit II is very sharp, followed by digit III. The law mark of digit IV is indistinct. One metatarsophalangeal region trace can be seen, it is round, blunt and faintly defined, positioned in line with the axis of digit III. GP0101-1-T1 shows weak to moderate mesaxony (average 0.45 , ranging between 0.39 and $0.49, N=3$ ). All three toes of GP0101-1-T1 are deep, well defined and have a 'fleshy' appearance. Based on the length of the stride, we estimated a speed of $\sim 1.44 \mathrm{~m} / \mathrm{s}$ or $\sim 5.18 \mathrm{~km} / \mathrm{h}$ (Alexander 1976; Thulborn 1990). The relative stride length (SL/ $h, h=$ hip height) is 1.52 , implying that the animal was walking, not trotting or running.

Another trackway also consisting of three footprints, numbered GP0101-1-T2-R1-L2. GP0101-1-T2 tracks have a mean length of $17 \mathrm{~cm}$ and a $\mathrm{L} / \mathrm{W}$ ratio of 1.4 . One step $(48 \mathrm{~cm})$ is about 2.82 times the length of a single footprint. GP0101-1-T2 shows weak to moderate mesaxony (average 0.5 , ranging between 0.38 and $0.62, N$ $=2$ ). Digit II in L2 was covered by T1-R1, indicating an earlier time of trace making. Adopting the formula of Alexander (1976), the speed of GP0101-1-T2 is $\sim 0.82 \mathrm{~m} / \mathrm{s}$ or $\sim 2.95$ $\mathrm{km} / \mathrm{h}$. The relative stride length $(\mathrm{SL} / h)$ is 1.11 , implying that the animal was walking. All separated tracks are poorly preserved. The possibility of undertracks should not be excluded. Its overall dimensions, length/width ratio and mesaxony are basically the same as those of $\mathrm{T} 1$ and T3.

Comparisons and discussion: $\mathrm{By}$ their size $(<25$ $\mathrm{cm}$ pes length) and by the degree of mesaxony, the tridactyl tracks from Xinglongwan can be assigned to the ichnogenus cf. Eubrontes (Olsen et al. 1998). They are characterized by weak to moderate mesaxony, which is close to that of typical footprints of the ichno- or morpho-family Eubrontidae, Lull 1904 (0.37-0.58 in Eubrontes type; Lockley 2009). Xinglongwan specimens lack the key feature of Eubrontes, such as a distinct metatarsophalangeal pad trace posterior to digit II, such as the type specimens of Eubrontes AC 151 (Olsen et al. 1998). On the other hand, the size and interdigital divarication of digits II-IV of the Xinglongwan specimens are different from those conventional. And the Jurassic Eubrontes. Xinglongwan specimens are apparently discrepant form cf. Irenesauripus isp. in the same area (Xing et al., 2011), which show hallux impressions. Thus, we classify the specimens as cf. Eubrontes because of the limited and moderately preserved sample.

\section{Preservation}

Notably, all of these tridactyl theropod tracks showed flattening (widening), especially manifested by lateral extension at the end of digit III, which is consistent with the externalmorphological changes discussed in detail by Lockley and Xing (2015), which are associated 
with differential effects of overburden pressures on different lithologies. Since all tridactyl tracks on GP0101-1 show a similar phenomenon, it is likely that the two Velociraptorichnus tracks also have the same changes. This leads us to realize that both Xinglongwan Velociraptorichnus and $V$. sichuanensis from E'mei are flatter/wider or significantly flatter than Shandong Velociraptorichnus (Li et al, 2007) or Mujiaowu Velociraptorichnus (Xing et al., 2015) which are likely to be external-morphological variations. Another supporting evidence is the similar flattening of grallatorid tracks from E'mei, which were initially mistaken for iguanodonts tracks (Zhen et al., 1994). However, Koreanaornis and Minisauripus, also from the E'mei site, do not appear to have significant flattening, which may be related to their tiny size and requires more comparison and discussion in the future.

\section{Conclusions}

1. Although northeastern Guizhou Province is part of the Sichuan Basin, this is the first record of deinonychosaurian tracks in Guizhou Province.

2. The didactcyl (Velociraptorichnus) and tridactyl tracks (cf. Eubrontes) described here both occur in the Jiaguan Formation ichnofauna, again demonstrating the diversity of theropod tracks in the Sichuan Basin.

3. Velociraptorichnus records from Hebei Province in northern China, Shandong Province in eastern China, and the Sichuan Basin (Sichuan and Guizhou Provinces) in southwest China are reviewed, and their commonality is described, as well as their universality in the Barremian, which may suggest that some specific tracks morphology may provide better geological age information in the future.

4. The "flattening" Velociraptorichnus type specimen is probably an externalmorphological variation.

5. The cf. Eubrontes from Xinglongwan contribute to the known diversity of theropod tracks from the Jiaguan Formation and suggest a high prevalence of cf. Eubrontes and Eubrontes type in small and medium size.

\section{Acknowledgements}

This research was funded by the National Natural Science Foundation of China (No. 41772008).

\section{References}

Alexander, R. (1976). Estimates of speeds of dinosaurs. Nature, 261, 129-130. https://doi.org/10.1038/261129a0

CGCMS (Compiling Group of Continental Mesozoic Stratigraphy and Palaeon-tology in Sichuan Basin of China). (1982). Continental Mesozoic Stratigraphyand Palaeontology in Sichuan Basin of China (pp.405). People's Publishing House of Sichuan Chengdu.

Geng, Q. (2011). Tectonic sequence stratigraphy and lithofacies paleogeography of Cretaceous-Paleogene in Sichuan Basin of China. Journal of Chengdu University of Technology Science \& Technology Edition, 38(4), 394-401.

Hao, Y.C., Su, D.Y., Yu, J.X., Li, Y.G., Zhang W.P.\& Liu G.F. (2000). The Stratigraphic Lexicon of China: Cretaceous (in Chinese) (pp. 124). Beijing Geological Publishing House.

Kim, J.Y., Kim, K.S.\& Lockley, M.G. (2008). New didactyl dinosaur footprints (Dromaeosauripus hamanensis ichnogen. et ichnosp. nov.) from the Early Cretaceous Haman Formation, south coast of Korea. Palaeogeography, Palaeoclimatology, Palaeoecology, 262, 72-78.

https://doi.org/10.1016/j.palaeo.2008.02.0 $\underline{03}$

Kim, J.Y., Lockley, M.G., Woo, J.O.\& Kim, S.H. (2012). Unusual didactyl traces from the Jinju Formation (Early Cretaceous South Korea) indicate a new ichnospecies of Dromaeosauripus. Ichnos, 19, 75-83. https://doi.org/10.1080/10420940.2012.66 4054

Kuang, H.W., Liu, Y.Q., Wu, Q.Z., Cheng, G.S., Xu, K.M., Liu, H., Peng, N., Xu, H., Chen, J., Wang, B.H., Xu, J.L., Wang, M.W.\& Zhang, P. (2013). Dinosaur track sites and palaeogeography of the late early Cretaceous in Shuhe rifting zone of Shandong Province. Journal of Palaeogeography, 15 (4), 435-453.

Leng, Y.Y., Luo, B.J., Zhang, L.\& Yang, L. (2017). The Destructive Effect of Chishui Danxia Caused by Rock Body Temperature Stress and Differentiating Weathering Guizhou Geology, 2,123-127.

Li, R.H., Lockley, M.G., Makovicky, P.J., Matsukawa, M., Norell, M.A., Har-ris, J.D.\& Liu, M.W. (2007). Behavioural and faunal implications of Early Cretaceous deinonychosaur trackways from China. Naturwissenschaften, 95,185-191. http://dx.doi.org/10.1007/s00114-007- 
$\underline{0310-7}$

Lockley, M.G. (2009). New perspectives on morphological variation in tridactyl footprints: clues to widespread convergence in developmental dynamics. Geological Quarterly, 53, 415-432.

Lockley, M.G., Li, J.J., Li, R.H., Matsukawa, M., Harris, J.D.\& Xing, L.D. (2013). A review of the tetrapod track record in China, with special reference to type ichnospecies: implications for ichnotaxonomy and paleobiology. Acta Geologica Sinica (English edition), 87(1), 1-20. https://doi.org/10.1111/1755-6724.12026

Lockley, M.G.\& Xing, L.D. (2015). Flattened fossil footprints: implications for paleobiology. Palaeogeography, Palaeoclimatology, Palaeoecology, 426, 85-94.

https://doi.org/10.1016/j.palaeo.2015.03.0 $\underline{08}$

Long, K., Chen, H.D., Lin, L.B., Xu, S.L.\& Cheng, L.X. (2011). Cretaceous tectonic sequence and litho-paleogeographic evolution in the Sichuan Basin. Journal of Stratigraphy (in Chinese), 35(3), 328-336.

Lull, R.S. (1904). Fossil footprints of the JuraTrias of North America. Memoirs of the Boston Society of Natural History, 5, 461557.

Olsen, P.E., Smith, J.B., McDonald, N.C. (1998). Type material of the type species of the classic theropod footprint genera Eubrontes, Anchisauripus and Grallator (Early Jurassic, Hartford and Deerfield Basins, Connecticut and Massachusetts, U.S.A. Journal of Vertebrate Paleontology, $18 \quad$ (3), 586-601. https://doi.org/10.1080/02724634.1998.10 011086

Thulborn, T. (1990). Dinosaur Tracks. London: Chapman \& Hall.

Wang, D.J. (2020). Study on characteristics of red layer weathering in Danxia landform area, Chishui city (p.79). Master's thesis, Guizhou University.

Xing, L.D., Mayor, A., Chen, Y., Harris, J.D.\& Burns, M.E. (2011a). The Folklore of Dinosaur Trackways in China: Impact on Paleontology. Ichnos, 18(4), 213-220. https://doi.org/10.1080/10420940.2011.63 $\underline{4038}$

Xing, L.D., Harris, J.D., Gierliński, G.D., Wang, W.M., Wang, Z.Y.\& Li, D.Q. (2011b). Middle Cretaceous Non-avian Theropod trackways from the Southern Margin of the Sichuan Basin, China. Acta Palaeontologica Sinica, 50(4), 470-480.

Xing, L.D., Lockley, M.G., Marty, D., Klein, H., Buckley, L.G., McCrea, R.T., Zhang, J.P.,
Gierliński, G.D., Divay, J.D.\& Wu, Q.Z. (2013a). Diverse dinosaur ichnoassemblages from the Lower Cretaceous Dasheng Group in the Yishu fault zone, Shandong Province, China. Cretaceous Research, 45, 114-134. https://doi.org/10.1016/j.cretres.2013.07.0 09

Xing, L.D., Li, D.Q., Harris, J.D., Bell, P.R., Azuma, Y., Fujita, M., Lee, Y.\& Currie, P.J. (2013b). A new deinonychosaurian track from the Lower Cretaceous Hekou Group, Gansu Province, China. Acta Palaeontologica Polonica, 58(4), 723-730. https://doi.org/10.4202/app.2011.0115

Xing, L.D., Lockley, M.G., Yang, G., Xu, X., Cao, J., Klein, H., Persons,W.S.I.V., Shen, H.J.\& Zheng, X.M.(2015). Unusual deinonychosaurian track morphology (Velociraptorichnus zhangi $\mathrm{n}$. ichnosp.) from the Lower Cretaceous Xiaoba Formation, Sichuan Province, China. Palaeoworld, 24, 283-292. https://doi.org/10.1016/j.palwor.2015.04.0 04

Xing, L.D.\& Lockley, M.G. (2016). Early Cretaceous dinosaur and other tetrapod tracks of southwestern China. Science Bulletin, 61(13), 1044-1051. https://doi.org/10.1007/s11434-016-1093Z

Xing, L.D., Lockley, M.G.\& Zhang, J.P. (2016a). Early Cretaceous dinosaur and other tetrapod tracks of southwestern China. Ningbo: Ningbo Publishing House, 1-410.

Xing, L.D., Lockley, M.G., Marty, D., Klein, H., Yang, G., Zhang, J.P., Peng, G.Z., Ye, Y., Persons, W.S.I.V., Yin, X.Y.\& Xu, T. (2016b). A diverse saurischian (theropodsauropod) dominated footprint assemblage from the Lower Cretaceous Jiaguan Formation in the Sichuan Basin, southwestern China: A new ornithischian ichnotaxon, pterosaur tracks and an unusual sauropod walking pattern. Cretaceous Research, 60, 176-193. https://doi.org/10.1016/j.cretres.2015.12.0 05

Xing, L.D., Lockley, M.G., Guo, Y., Klein, H., Zhang, Junqiang, Zhang, J., Persons IV, W.S., Romilio, A., Tang, Y.\& Wang, X. (2018a). Multiple parallel deinonychosaurian trackways from a diverse dinosaur track assemblage of the Lower Cretaceous Dasheng Group of Shandong Province, China. Cretaceous Research, 90, 40-55. https://doi.org/10.1016/j.cretres.2018.04.0 $\underline{05}$

Xing, L.D., Lockley, M.G., Tang, Y.Z., Romilio, 
A., Xu, T., Li, X.W., Tang, Y.\& Li, Y.Z. (2018b). Tetrapod track assemblages from Lower Cretaceous desert facies in the Ordos Basin, Shaanxi Province, China, and their implications for Mesozoic paleoecology. Palaeogeography, Palaeoclimatology, Palaeoecology, 507, 114.

https://doi.org/10.1016/j.palaeo.2018.05.0 $\underline{16}$

Xing, L.D., Lockley, M.G., Du, T.M., Zhang, L.J., Klein, H., Romilio, A., Persons, W.S.IV., Wang, K., Li, Z.Y.\& Wan, X.Q. (2019).
Dinosaur tracks from the JurassicCretaceous boundary Tuchengzi Formation (Hebei Province, China) used as building stones in the Chengde imperial summer resort: age, ichnology, and history. Cretaceous Research, 107, 104310. https://doi.org/10.1016/j.cretres.2019.104 310

Zhen, S.N., Li, J.J., Chen, W.\& Zhu, S. (1994). Dinosaur and bird footprints fromthe Lower Cretaceous of Emei County Sichuan. Memoirs of the Beijing Natural History, 54, 105-120.

Publisher's note: Eurasia Academic Publishing Group (EAPG) remains neutral with regard to jurisdictional claims in published maps and institutional affiliations.

Open Access This article is licensed under a Creative Commons Attribution-NoDerivatives 4.0 International (CC BY-ND 4.0) licence, which permits copy and redistribute the material in any medium or format for any purpose, even commercially. The licensor cannot revoke these freedoms as long as you follow the licence terms. Under the following terms you must give appropriate credit, provide a link to the license, and indicate if changes were made. You may do so in any reasonable manner, but not in any way that suggests the licensor endorsed you or your use. If you remix, transform, or build upon the material, you may not distribute the modified material.

To view a copy of this license, visit https://creativecommons.org/licenses/by-nd/4.0/. 